\title{
Massive star disks
}

\author{
Qizhou Zhang \\ Harvard-Smithsonian Center for Astrophysics \\ 60 Garden Street \\ Cambridge, MA 02138 \\ USA \\ email: qzhang@cfa.harvard.edu
}

\begin{abstract}
Recent years have seen accumulating evidence for infall and rotation around disk like structures toward young stars of spectral types of early B and possibly late O. Observations indicate that the disks or tori are massive in comparison with the mass of the central stars and geometrically thick. Both properties distinguish them from the disks around low mass stars, in particular T-Tauri type stars.

I review observational techniques used in studying disks around massive young stars and summarize properties of disks derived from observations. I will discuss implications of these findings for the formation of massive stars.
\end{abstract}

Keywords. Accretion, accretion disks, star: formation, ISM: HII region

\section{Introduction}

Protostars radiate its gravitational energy in the Kelvin-Helmholtz time scale. For massive protostars with $\mathrm{M}>8 \mathrm{M}_{\odot}$, the Kelvin-Helmholtz time scale is shorter than the free-fall time scale of dense cores. Therefore, a star may reach the main sequence while the matter in its natal molecular core still undergoes dynamic collapse. Detailed stellar evolution calculations confirm that protostars more massive than $8 \mathrm{M}_{\odot}$ do not have a pre-main sequence phase, but evolve directly to the main sequence phase (Stahler 1980; Palla \& Stahler 1993; Palla this Proceedings).

Once massive stars reach the main sequence, strong radiation in the ultra violet and optical wavelengths interacts with dusty cores and exerts a pressure on the infalling material. This radiation problem has been investigated over many years (Larson 1969; Larson \& Starrfield 1971; Kahn 1974; York \& Krügel 1977; Wolfire \& Cassinelli 1987; Osorio, Lizano \& D'Alessio 1999). The condition that the radiation force balances the gravitational force, i.e. $\frac{\kappa L}{4 \pi c} \sim G M$, gives the maximum stellar mass of $\sim 10 \mathrm{M}_{\odot}($ Wolfire \& Cassinelli 1987).

This mass limit can be increased, as argued by Kahn (1974) and Wolfire \& Cassinelli (1987), if the mass infall rate in the envelope exceeds $10^{-5} \mathrm{M}_{\odot} \mathrm{yr}^{-1}$. The effect of radiation on the infalling matter is mostly on the thin layer where dust is sublimated. Inside the radius of dust sublimation, dust is distroyed. The opacity is greatly reduced. Outside the dust destruction radius, stellar radiation is absorbed and re-emitted mostly in the infrared wavelengths. The dust opacity is again reduced. If the infalling matter carries enough momentum, it can penetrate the thin layer at the dust sublimation radius, allowing infall to be continued. This criteria requires $\dot{M} V \gtrsim \frac{L}{C}$, where $\dot{M}$ is the mass infall rate, $\mathrm{L}$ the total luminosity due to stellar radiation and accretion, and $\mathrm{V}$ the infall velocity. Therefore, a large mass infall rate is crucial for continuous accretion and formation of more massive stars. 
The problem with radiation pressure inspired one view of massive star formation. In a model proposed by Bonnell et al. (1998) and Bonnell \& Bate (2002), massive stars form via coalescence of low- to intermediate-mass protostars. This model circumvents the radiation pressure problem by forming lower mass stars first and then merging them together to make a massive star. The model accounts for the high rate of multiplicity in massive stars, but requires exceedingly high stellar density $\left(\gtrsim 10^{7} p c^{-3}\right)$ in order for merger to be effective. In addition, it is not clear how to dissipate the kinetic and gravitational energy during the merging process (Bally \& Zinnecker 2005).

The other view of massive star formation builds upon the knowledge of low-mass star formation. This model argues that massive stars form via disk accretion with high accretion rates (Norberg \& Maeder 2000; McKee \& Tan 2003; Keto 2003).

The presence of a disk near massive young star can significantly reduce the effect of radiation on the infalling material by reducing the exposure of matter to the radiation field and by allowing photons to escape along the polar axis (Nagano 1989; Jijina \& Adams 1996; Yorke \& Sonnhalter 2002; Krumholtz, McKee \& Klein 2005). For example, the criterion on momentum balance discussed above is modified: $\frac{4 \pi}{\Omega} \dot{M} V \gtrsim \frac{L}{C}$, where $\Omega$ is the solid angle of the disk subtended with respect to the star. Since $\Omega<4 \pi$, the maximum luminosity allowed is increased significantly in the presence of a disk.

It is clear from theoretical considerations that disk is a key component in understanding the formation process of massive stars. In the past few years, progress has been made from the observational side in identifying flatten disk-like structures toward massive young stars. In this article, I will review the observational evidence for disk and summarize disk properties. I will discuss the implication of these findings for massive star formation.

\section{Identification of Disks around Massive Stars}

Stars of $1 \mathrm{M}_{\odot}$ spend $10^{7}$ yrs at the pre-main sequence stage before reaching the main sequence track. In contrast, massive stars do not have the pre-main sequence phase (Stahler 1980; Palla \& Stahler 1993; Palla, this Proceedings). Because of rapid evolution, dense molecular cores around massive young stars do not have enough time to dissipate molecular gas before the UV radiation from the main-sequence star ionizes the immediate surroundings. This situation hampers the search for disks around massive young stars. Since disks, if exist, are always deeply embedded in the dense natal molecular cloud cores, signatures of disks are mixed with those from the core. Discerning the signature in the presence of competing processes such as infall and outflows is inherently difficult, even at high angular resolution. In a similar situation, there is a lack of clean examples of disks toward the deeply embedded CLASS 0 and I low-mass stars. In case of HL Tau - a CLASS I low-mass object, for example, three interpretations (disk, disk plus infall, and outflow) were offered by different groups (Sargent \& Beckwith 1987; Hayashi et al. 1993; Cabrit et al. 1996). The best example of Keplerian disks surrounding low- to intermediate-mass stars comes from the more evolved objects such as T Tauri or Herbig Ae/Be stars (e.g. Dutrey et al. 1996; Mannings \& Sargent 1997). Massive disks are always surrounded by dense molecular gas, similar to the situation in CLASS 0 and I objects of low-mass stars. In addition, massive stars are in general found at larger distances, the presence of other stars in the cluster environment, and relatively small number of massive young stars make it more difficult in the disk search. These factors have all contributed to the lack of progress in this field.

Various techniques and a variety of tracers have been used in search for disks toward massive young stars. Because of the large distances of massive young stars and small size scales of disks, high angular resolution is required. 
Disks have been sought in both thermal and non-thermal emission. The $\mathrm{H}_{2} \mathrm{O}$ maser transition at $22 \mathrm{GHz}$ and $\mathrm{CH}_{3} \mathrm{OH}$ masers at $6 \mathrm{GHz}$ are often used in search for disks toward massive stars (Torrelles et al. 1998; Norris et al. 1998; Phillips et al. 1998). Toward the Cepheus A region, Torrelles et al. (1998) reported a cluster of maser emission associated with a young star with spectral type of early B. At an angular resolution of $0^{\prime \prime} .1$, Torrelles et al. found the maser spots distributed in a $1^{\prime \prime} \times 2^{\prime \prime}$ region with the major axis perpendicular to the ionized jet. The radial velocity of the maser spots is consistent with a rotation pattern. With follow-up observations with the VLBA, the maser spots are resolved into a series of filaments, which does not support the earlier interpretation of a rotating disk (Torrelles et al. 2001).

Similarly, Norris et al. (1998) found many ultra compact HII (UCHII) regions coincident with $\mathrm{CH}_{3} \mathrm{OH}$ maser spots distributed in a linear morphology with a velocity gradient. Norris et al. suggest that $\mathrm{CH}_{3} \mathrm{OH}$ masers trace disk around newly formed massive stars. The number of linear structures in the $\mathrm{CH}_{3} \mathrm{OH}$ maser indicates that the fraction of edge-on disks exceeds the faction in a sample of randomly oriented disks. This raises concerns on the disk interpretation. However, Norris et al. argued that the long pass length required for maser amplification conspires the selection effect: Only the edge-on disks have the long path length for maser emission. However, Walsh et al. (1998) suggest that $\mathrm{CH}_{3} \mathrm{OH}$ masers are associated with individual stars in the cluster.

Other masers, such as $\mathrm{OH}, \mathrm{SiO}$ and hydrogen recombination emission have also been associated with disks. Wright et al. (1995) reported an $\mathrm{SiO} \mathrm{J}=2-1 \mathrm{~V}=1$ maser toward the Orion KL region. The linear structure in the $\mathrm{SiO}$ emission is interpreted as a disk surrounding a massive star. The subsequent observations with VLBA by Greenhill et al. (2002) found that the proper motion of the $\mathrm{SiO}$ masers are consistent with an expanding disk around an early B stars. The hydrogen recombination lines are rarely seen in maser action. However, toward the unique young stellar object MWC 349, maser emission is seen in the hydrogen recombination lines higher than $100 \mathrm{GHz}$ (Martín-Pintado et al. 1989). The maser lines show double horn profiles which can be modeled with disk origin (Thum et al. 1994).

Maser emission requires special conditions for population inversion, thus, does not necessarily trace the bulk of molecular gas. Reports of disk detections in maser emission do not escape controversy. Some of the claims have been refuted by higher angular resolution studies. For detailed reviews on this subject, see contributions in this proceedings (Burton; De Buizer; Sobolev; Torrelles, this Proceedings).

In general, thermal emission is the preferred tracer of disks. The gas and dust in the inner part of the disk are heated to a few $1000 \mathrm{~K}$ and radiate mostly in the near infrared wavelengths. For low-mass young stars, the band head of the CO overtone at $2.4 \mu \mathrm{m}$ reveals line profiles that can be fitted with rotation (e.g. Carr 1987). This technique has been extended to massive young stars. The CO lines from some UCHII regions can be fitted with Keplerian motion, possibly from a disk (Blum, this Proceedings). In addition, the advent of infrared interferometers shows promise of imaging the inner edge of disks around Herbig Ae/Be stars (Eisner et al. 2004; Monnier et al. 2005).

Thermal emission from gas and dust in cores of 10 to a few $100 \mathrm{~K}$ emits mostly in the millimeter to far infrared wavelengths. Thus, radio and submm interferometers are key tools in deciphering disks in deeply embedded objects. At these wavelengths, emission arises from the outer part of the disk and possibly the inner part of the envelope. Since disk and evelope cannot be separated easily through observations, I refer to these entities as disk-like structures or Tori. In the following section, I will review disk detections toward $\mathrm{B}$ and $\mathrm{O}$ type stars using these techniques. 


\section{Evidence for Disks around Massive Young Stars}

\subsection{Disks around early B-type Stars}

There is growing evidence that disks are present and might be even common in young stellar objects of spectral type of early B. The direct imaging of disk-like structures in both (sub)mm continuum and molecular lines is increasing in number (AFGL490: Schreyer 2002, $\mathrm{GH}_{2} \mathrm{O}$ 092.67+03.07: Bernard et al. 1999, M17: Chini et al. 2003; Sako et al. 2005, G192.16-3.82: Shepherd et al. 1998; Shepherd \& Kurtz 1999; Shepherd, Claussen \& Kurtz 2001, AFGL5142: Hunter et al. 1999; Zhang et al. 2002; IRAS20126+4104: Cesaroni et al. 1997, 1999; Zhang et al. 1998, 1999; Cesaroni et al. 2005, NGC7538: Sandell et al. 2003; IRAS18089-1732: Beuther et al. 2004; 2005).

In addition to direct imaging of disk-like structures, surveys of molecular outflows provide indirect evidence for disks in massive young stars (Shepherd, this Proceedings). In the past 10 years, there has be a number of surveys for molecular outflows toward regions of massive star formation (Beuther et al. 2002; Zhang et al. 2001, 2005; Osterloh, Henning \& Launhardt 1997; Shepherd \& Churchwell 1996; McCutcheon et al. 1991; Wilking et al. 1989; Snell et al. 1988, 1990). A large fraction ( 90\%) of massive star forming regions have massive molecular outflows (Zhang et al. 2000; 2005; Beuther et al. 2002). These surveys are conducted with single dish telescopes with angular resolutions of $20^{\prime \prime}-30^{\prime \prime}$ (Wu et al. 2005). Thus, identifying the driving source is difficult. However, Zhang et al. (2005) argue that the massive outflows from the survey are powered by the more massive stars in the region rather than the less massive stars in the cluster. Most objects in these surveys have far infrared luminosities of $10^{4} \mathrm{~L}_{\odot}$, corresponding to a single zero-age-main-sequence star of B. If disks are connect to accretion, as in the case of low-mass stars, these studies of molecular outflows provide indirect evidence that disks are common around massive stars.

Among all the massive star disks reported, IRAS 20126+4104 is perhaps the best example. The object is an extremely young high-mass star at a distance of $1.7 \mathrm{kpc}$ (Wilking et al. 1989). Its far infrared luminosity of $1.3 \times 10^{4} \mathrm{~L}_{\odot}$ corresponds to a single zero-age-main-sequence star of B0.5 (Cesaroni et al. 1997). VLA observations at 6, 3.6, 2 and $1.3 \mathrm{~cm}$ (Wilking et al. 1989; Tofani et al. 1995; Cesaroni et al. 1997) have only produced a positive detection of $0.3 \mathrm{mJy}$ at $3.6 \mathrm{~cm}$. Cesaroni et al. $(1997 ; 1999)$ found a compact bipolar outflow in the $\mathrm{HCO}^{+}$(1-0) line and $\mathrm{SiO}(2-1)$, embedded in a larger scale bipolar CO (1.35 pc) outflow (Wilking, Blackwell, \& Mundy 1990; Shepherd et al. 2000). At the geometric center of the SiO jet, Cesaroni et al. $(1997 ; 1999)$ and Zhang et al. $(1998 ; 1999)$ found a compact disk-like structure in $\mathrm{NH}_{3}(1,1),(2,2)$ and $(3,3)$, the $\mathrm{CH}_{3} \mathrm{CN}$ (5-4) and (12-11) lines, and $\mathrm{C}^{34} \mathrm{~S}$ associated with the $\mathrm{mm}$ continuum source. All the core tracers reveal an elongated compact emission perpendicular to the axis of the $\mathrm{SiO}$ jet. The velocity field along the major axis of the disk can be modeled with a Keplerian rotation. Figure 1 shows the structure and kinematics in the disk in the $\mathrm{NH}_{3}$ $(1,1)$ and $(2,2)$ lines and the SiO jet (Zhang et al. 1997; Cesaroni et al. 1999)

\subsection{Disks/Tori around O-type Young Stars}

Because of the smaller number in the Galaxy and more rapid prestellar evolution, O-type stars are difficult to find, especially at the early phases of evolution. Therefore, compelling cases for disk-like structures remain to be made. However, signature of rotation has been reported toward (proto)stellar candidate IRAS18089-1732 (Beuther et al. 2004; 2005). and more luminous objects such as G24.78, G28-0.05, G31.41+0.31, and G10.6 (Beltran et al. 2004, 2005; Sollins et al. 2005a; Keto, Ho \& Haschick 1987; Sollins et al. 2005b; Sollins \& Ho 2005) where UCHıI regions has already emerged. 



Figure 1. Structures of compact, rotating disk - integrated emissions of $\mathrm{NH}_{3}(1,1)$ and $(2,2)$ transitions towards IRAS 20126+4104, obtained from VLA (Zhang et al. 1998), as compared to the bipolar $\mathrm{SiO}$ jet (Cesaroni et al 1999).

IRAS18089-1732 is a young massive star with a far infrared luminosity of $6 \times 10^{4}$ $\mathrm{L}_{\odot}$ (Sridharan et al. 2002). Millimeter observations reveal a massive core of more than $2000 \mathrm{M}_{\odot}$ and $\mathrm{H}_{2} \mathrm{O}$ and $\mathrm{CH}_{3} \mathrm{OH}$ maser emission (Beuther et al. 2002). Despite of high luminosity, only a weak radio continuum of $1 \mathrm{mJy}$ is detected at $3.6 \mathrm{~cm}$ (Beuther et al. 2002). Recent studies with the Submillimeter Array resolved a unipolar outflow in the $\mathrm{SiO}$ 5-4 transition toward the northeast direction. Evidence of rotation is found in the $\mathrm{HCOOCH}_{3}$ lines in form of velocity gradient, roughly perpendicular to the direction of molecular outflow (Beuther et al. 2004; 2005). The mass in the disk is estimated to be about $20 \mathrm{M}_{\odot}$ with a diameter of $1800 \mathrm{AU}$. Continuum emissions at $1 \mathrm{~mm}$ and $0.8 \mathrm{~mm}$ show one dominant peak in dust emission, possibly from the most massive star in the region. If we convert the luminosity to stellar mass using the prestellar calculation by Beech \& Mihalas (1994), we obtain a mass of $23 \mathrm{M}_{\odot}$. This corresponds to a star of spectral type of O9. It is likely that the star is still in an active accretion phase based on the presence of strong molecular outflow. If this is true, the final mass of the star will be higher after the accretion terminates.

G24.78+0.08 consists of four continuum peaks at $2 \mathrm{~mm}$ (Furuya et al. 2002) with a far infrared luminosity of $7.9 \times 10^{5} \mathrm{~L}_{\odot}$ at a distance of $7.9 \mathrm{kpc}$. Sources A and B coincide with emission at $1.3 \mathrm{~cm}$ (Codella, Testi \& Cesaroni 1997). At higher angular resolution, source A is resolved into two cores A1 and A2 at 1.4mm (Beltran et al. 2004; 2005) and $\mathrm{CH}_{3} \mathrm{CN}$. A velocity gradient is found toward both $\mathrm{A} 1$ and $\mathrm{A} 2$, perpendicular to a $\mathrm{CO}$ outflow in the northwest-southeast direction. The mass in the disk-like structure is 160 $\mathrm{M}_{\odot}$ with a diameter of $12,000 \mathrm{AU}$.

Of all the massive stars with disks reported, G10.6 may represent a different mode of star formation. The UCHII region G10.6-0.4, at a distance of $6.0 \mathrm{kpc}$, is the brightest member of a complex of various compact HiI regions (Wood \& Churchwell 1989) in an area of active star formation. The associated IRAS point source, IRAS 18075-1956, has 
a luminosity of $9.2 \times 10^{5} \mathrm{~L}_{\odot}$ (Casoli et al. 1986). Studies of the inversion lines of $\mathrm{NH}_{3}$ have determined that the molecular core is in a disk/torus rotating and collapsing inward toward the UCHII region (Keto et al. 1987), excited by a cluster of stars of $300 \mathrm{M}_{\odot}$ (Keto 2002; Sollins et al. 2005b; Sollins \& Ho 2005). At the highest resolution ( $\left.0^{\prime \prime} .1\right)$ achieved, infall and rotation in the molecular gas were seen simultaneously in absorption, showing that the molecular gas was spiraling inward on size scales comparable to the size of the UCHII region (Sollins et al. 2005; Sollins \& Ho 2005). Unlike the disk/torus toward other objects, the accretion in G10.6 is seen toward a cluster of stars.

\section{Disk Characteristics}

Table 1 lists a subset of disks/tori with measured mass, size and mass infall rate. Here, the infall rate is defined as the mass influx within a certain sphere. Because of the wind and outflows, not all the infalling mass will reach the central star. Thus, the infall rate is the upper limit of the accretion rate. For low-mass stars, the mass ejected in the wind amounts to less than one-third of the infalling matter (Shu et al. 1991), thus the infall rate is comparable to the accretion rate. We will not distinguish infall rate and accretion rate in the following discussions.

Table 1. Disk Parameters

\begin{tabular}{|c|c|c|c|c|}
\hline $\begin{array}{l}\text { Source } \\
\text { Name }\end{array}$ & $\begin{aligned} \mathrm{Lum} \\
\mathrm{L} \odot\end{aligned}$ & $\begin{array}{r}M_{\text {disk }} \\
\mathrm{M}_{\odot}\end{array}$ & $\begin{array}{r}D_{\text {disk }} \\
\text { AU }\end{array}$ & $\begin{array}{l}\text { Accretion rate } \\
\mathrm{M}_{\odot} \mathrm{yr}^{-1}\end{array}$ \\
\hline G192 ${ }^{1}$ & $3 \mathrm{e} 3$ & 15 & 1000 & $1 \mathrm{e}-4$ \\
\hline AFGL5142 2 & $3 \mathrm{e} 3$ & 4 & 1800 & $4 e-5$ \\
\hline $\mathrm{I} 20126^{3,4}$ & $1.3 \mathrm{e} 4$ & 5 & 1600 & $9.8 \mathrm{e}-4$ \\
\hline $\mathrm{I} 18566^{5}$ & $6 \mathrm{e} 4$ & 60 & 8000 & $1.6 \mathrm{e}-3$ \\
\hline G28.20 & $1 \mathrm{e} 5$ & 20 & 6000 & $5 e-3$ \\
\hline G24.78 & $7 \mathrm{e} 5$ & 160 & 6000 & $9 \mathrm{e}-3$ \\
\hline G10. $6^{8}$ & $9 \mathrm{e} 5$ & 300 & 12000 & $2 \mathrm{e}-2$ \\
\hline
\end{tabular}

1: Shepherd et al. 2001; 2: Zhang et al. 2002; 3: Cesaroni et al. 1999; 4: Zhang et al. 1998; 5: Zhang et al. in preparation; 6: Sollins et al. 2005a; 7: Beltran et al. 2004; Sollins et al. 2005b

Despite the uncertainty in the measurements, disk mass, sizes and accretion rate in Table 1 all increase with the increase of luminosity. This is not simply a resolution effect due to large distances to more luminous objects, since the linear resolution achieved for two of the most luminous objects (G28.20 and G10.6) are comparable to the less luminous ones in the sample with closer distances.

The sizes of the disk-like structures in Table 1 are typically a few 1000 AU with masses of a few to a few tens of $\mathrm{M}_{\odot}$. As mentioned before, these objects are still deeply embedded, it is difficult to separate the outer edges of the disk from the envelope. The sizes can be over estimated. In G192, where the highest linear resolution is achieved in dust continuum, a disk size of about $100 \mathrm{AU}$ is reported (Shepherd et al. 2001). In the molecular gas, some of the tracers are probably optically thick, thus, increasing angular resolution may not reduce the disk size. Likewise, the disk masses measured here are also large. Using the mass-luminosity relation from calculations of prestellar evolution (e.g. Beech \& Mihalas 1994), we can convert luminosity to stellar mass. For G192, we obtain a mass of $10 \mathrm{M}_{\odot}$, comparable to the mass in the disk. This trend continues similarly for more luminous objects.

It is also worth noting that the accretion rates measured are in the range of $10^{-4}-10^{-2}$ $\mathrm{M}_{\odot} \mathrm{yr}^{-1}$. The typical time scales needed to form these stars are $10^{5} \mathrm{yrs}$ : The high 
accretion rates are consistent with the short time scale needed for massive star formation. Further more, the high accretion rates are also in line with the requirement for infall to overcome the radiation pressure.

We find a tight correlation between the accretion rate vs. the luminosity of the region. The least Squared fit yields a relation of

$$
\dot{M}\left(M_{\odot}\right)=5 \times 10^{-8}\left(\frac{L}{L_{\odot}}\right)^{0.94},
$$

with a correlation coefficent of $96 \%$. We note that correction of the luminosity due to infall does not change the relation. If we convert luminosity to the stellar mass using the prestellar evolution calculation (Beech \& Mihalas 1994), we again find a tight correlation between the mass accretion rate versus the stellar mass, as shown in Figure 2. For G10.6, the stellar mass infered from luminosity is a lower limit. A Least Squared fit yields

$$
\dot{M}\left(M_{\odot}\right)=1.6 \times 10^{-6}\left(\frac{M}{M_{\odot}}\right)^{2.2} .
$$

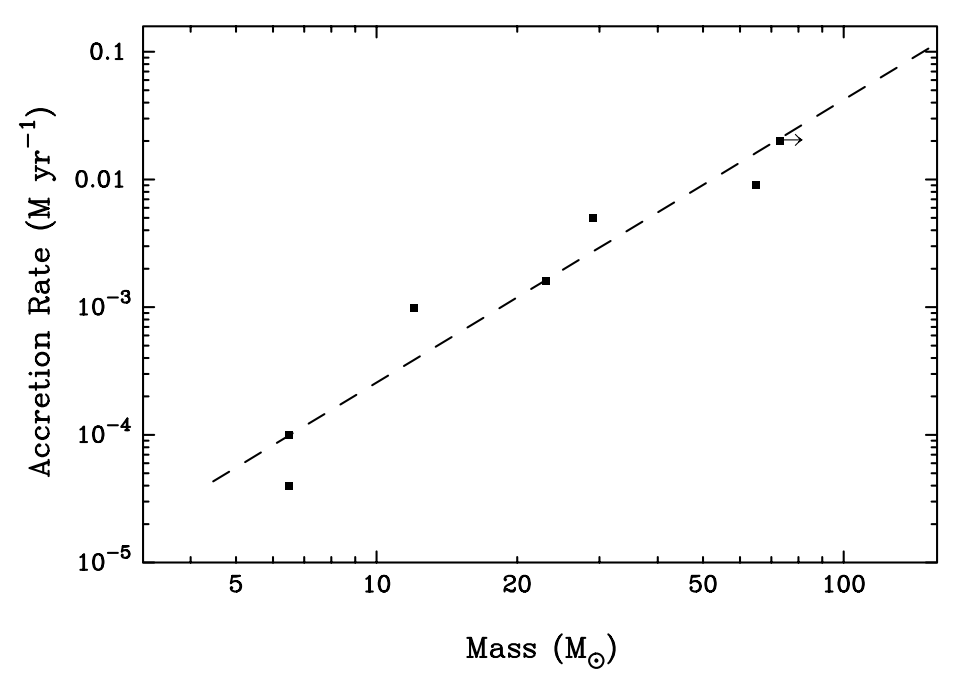

Figure 2. The mass accretion rate versus the mass of the central star.

\section{Implication for Massive Star Formation}

The direct detection of disk surrounding $\mathrm{B}$ and late $\mathrm{O}$ type stars indicates an accretion process of massive star formation. The high accretion rate further suggest that infalling matter can overcome the radiation pressure. In evolution calculations of massive star formation (Beech \& Mihalas 1994; Norberg \& Maeder 2000; Keto 2003), it is assumed that the accretion rate increases stellar mass.

\subsection{Accretion through Turbulent Core}

How can this accretion rate be achieved? In low mass cores, the accretion rate in an isolated isothermal sphere follows

$$
\dot{M}=\frac{a C_{s}^{3}}{G}
$$


where $C_{s}$ is the sound speed, a a constant of unity (Shu, Adam \& Lizano 1987). For typical conditions $(10 \mathrm{~K})$ in the low-mass cores, this gives an accretion rate of $1.5 \times 10^{-6}$ $\mathrm{M}_{\odot} \mathrm{yr}^{-1}$. However, in massive star forming cores, the line widths are normally much larger than the sound speed (Caselli \& Myers 1995). It has been argued that in these cores, the above accretion rate is still valid, but the thermal sound speed $C_{s}$ is modified (Nagano 1989; McKee \& Tan 2002, 2003):

$$
\dot{M}=\frac{a\left(C_{s}^{2}+V_{t}^{2}+V_{B}^{2}\right)^{3 / 2}}{G},
$$

where $V_{t}$ is the turbulent velocity and $V_{B}$ the velocity related to magnetic fields. For a turbulent line width of a few $\mathrm{km} \mathrm{s}^{-1}$, the accretion rate can be increased to $10^{-4} \mathrm{M}_{\odot} \mathrm{yr}^{-1}$.

The empirical relation in Figure 2 gives an accretion rate that scales as $M^{2.2}$, similar to the Bondi-Hoyle accretion (Bondi \& Hoyle 1944). However, as McKee \& Tan (2003) pointed out, Bondi-Hoyle accretion is not effective and the magnitude of the accretion rates are too low as compared to the measurements.

\subsection{Disk Instabilities and Angular Momentum Dissipation}

It appears that the disk-like structures observed toward massive young stars are rather massive as compared to the mass of the stars. The self gravity of the disk can play an important role in disk dynamics. Instability in disks is determined by the parameter $Q$ (Toomre 1964), defined as

$$
Q=\frac{C_{s} \kappa}{\pi G \Sigma}
$$

Here $\kappa$ is the epicycle frequency, $\Sigma$ is the surface density of the disk. When $Q<1$, disk is subjected to gravitational instability and produces spiral structures. Such structure is widely observed in galaxies, and has been recently reported toward disks surrounding young stars (e.g. AB Aurige: AFGL490: Schreyer et al. 2002). In simulations by Ladato \& Rice (2004), spiral structures develop in disks and are effective in transporting angular momentum and energy outwards. Efficient angular momentum loss in disks around massive stars is essential for high accretion rates observed toward massive stars.

\section{Summary and Future Prospective}

Our knowledge on massive star birth has advanced significantly in the last ten years: There is mounting evidence that early B stars form via disk accretion and mass ejection in form of molecular outflows. The observed accretion rates are as high as $10^{-3} \mathrm{M}_{\odot} \mathrm{yr}^{-1}$. It is conceiverable that some of the early B stars will evolve into late $\mathrm{O}$ type stars when finishing accreting. For more luminous O-type stars, the picture is less clear. Limited observations suggest that late O-type stars also has disk-like structures and powers molecular outflows. However, in more massive O-type stars, for example in G10.6, the infall and accretion is seen in a disk-like structure toward a cluster.

Understanding the formation of the most massive $O$ stars remains to be a challenging task. The knowledge on the formation process of these objects will bridge the gap between the typical Galactic star foramtion and star burst phenomena. Observationally, it is important to focus the research on the more massive young stellar objects, in particular, objects with no detectable $\mathrm{cm}$ emission or those associated with hyper compact HII regions (Kurtz, this Proceedings). Studying of these regions will yield fruitful kinematic information regarding the formation process of the more massive $\mathrm{O}$ stars. 


\section{References}

Bally, J. \& Zinnecker, H. 2005, AJ, in press

Beech, M. \& Mihalas, R. 1994, ApJS, 95, 517

Beltrán, M.T., Cesaroni, R., Neri, R., Codella, C., Furuya, R.S., Testi, L., \& Olmi, L. 2004, ApJ 601, L187

Beltrán, M.T., Cesaroni, R., Neri, R., Codella, C., Furuya, R.S., Testi, L., \& Olmi, L. 2005, $\mathrm{A} \& \mathrm{~A}$ in press

Bernard, J.P., Dobashi, K., \& Momose, M. 1999, A\&A 350, 197

Beuther H., Hunter, T.R., Zhang, Q., Sridharan, T.K., Zhao, J.-H., Sollins, P., Ho, P.T.P., Ohashi, N., Su, Y.N., Lim, J., \& Liu, S.-Y. 2004, ApJ 616, L23

Beuther, H., Schilke, P., Sridharan, T.K., Menten, K.M., \& Wyrowski, F., 2002, A\&A, 383, 892

Beuther, H., Zhang, Q., Sridharan, T.K., \& Chen, Y. 2005, ApJ, accepted

Bondi, H. \& Hoyle, F. 1944, MNRAS, 104, 273

Bonnell, I.A. \& Bate, M.R. 2002, MNRAS 336, 659

Bonnell, I., Bate, M.R., \& Zinnecker, H. 1998, MNRAS, 298, 93

Cabrit, S., Guilloteau, S., Andre, P., Bertout, C., Montmerle, T., \& Schuster, K. A\&A, 305, 527

Carr, J.S. 1987, ApJ, 323, 170

Caselli, P. \& Myers, P.C. 1995, ApJ, 446, 665

Cesaroni, R., Felli, M., Testi, L., Walmsley, C.M., \& Olmi, L. 1997, A\&A 325, 725

Cesaroni, R., Hofner, P., Walmsley, C.M., \& Churchwell, E. 1998, A\&A 331, 709

Cesaroni, R., Felli, M., Jenness, T., Neri, R., Olmi, L., Robberto, M., Testi, L., \& Walmsley, C.M. 1999, A\&A 345, 949

Cesaroni, R., Neri, R., Olmi, L., Testi, L., Walmsley, C.M., \& Hofner, P. 2005, A\&A 434, 1039

Chini, R., Hoffmeister, V., Kimeswenger, S., Nielbock, M., Nürnberger, D., Schmidtobreick, L., \& Sterzik, M. 2004, Nature 429, 155

Casoli, F., Combes, F., Dupraz, C., Gerin, M., \& Boulanger, F. 1986, A\&A, 169, 281

De Buizer, J.M. 2003, MNRAS 341, 277

Dutrey, A., Guilloteau, S., Duvert, G., Prato, L., Simon, M., Schuster, K., \& Menard, F. 1996, A\&A, 309, 493

Eisner, J.A., Lane, B.F., Hillenbrand, L.A., Akeson, R.L., \& Sargent, A.I. ApJ, 613, 1049

Garay, G. \& Lizano, S. 1999, PASP, 111, 1049

Greenhill, L.J., Reid, M.J., Chandler, C.J., Diamond, P.J., \& Elitzur, M. 2004, in Star Formation at High Angular Resolution, ASP Conference Series, IAU Symposium, 221, 155

Hayashi, M., Ohashi, N., \& Miyama, S.M. 1993, ApJ, 418, L71

Hunter, T.R., Testi, L., Zhang, Q., \& Sridharan, T.K. 1999, AJ, 118, 477

Jijina, J. \& Adams, F.C. 1996, 462, 874

Kahn, F.D. 1974, A\&A, 37, 149

Keto, E. 2003, ApJ, 599, 1196

Keto, E.R., Ho, P.T.P., \& Haschick, A.D. 1988, ApJ 324, 920

Krumholtz, M.R., McKee, C.F., \& Klein, R.I. 2005, ApJ, 618, L33

Larson, R.B. 1969, MNRAS, 145, 271

Larson, R.B. \& Starrfield, S. 1971, A\&A, 13, L190

Lodato, G. \& Rice, W.K.M. 2005, MNRAS 358, 1489

Mannings, V., Sargent, A.I. 1997, ApJ, 490, 792

Martín-Pintado, J., Bachiller, R., Thum, C., \& Walmsley, M. 1989, A\&A, 215, L13

McCutcheon W.H., Dewdney, P.E., Purton, R., \& Sato, T. 1991, AJ, 101, 1435

McKee, C.F. \& Tan, J.C. 2002, Nature, 416, 59

McKee, C.F. \& Tan, J.C. 2003, ApJ, 585, 850

Minier, V., Booth, R.S., \& Conway, J.E. 1998, A\&A 336, L5

Minier, V., Booth, R.S., \& Conway, J.E. 2000, A\&A 362, 1093

Monnier, J.D. et al. 2005, ApJ, 624, 832

Nagano, T. 1989, ApJ, 345, 464

Norberg, P. \& Maeder, A. 2000, A\&A, 359, 1025 
Norris, R.P., Byleveld, S.E., Diamond, P.J., Ellingsen, S.P., Ferris, R.H., Gough, R.G., Kesteven, M.J., McCulloch, P.M., Phillips, C.J., Reynolds, J.E., Tzioumis, A.K., Takahashi, Y., Troup, E.R., \& Wellington, K.J. 1998, ApJ 508, 275

Osorio, M., Lizano, S., \& D'Alessio, D. 1999, ApJ, 525, 808

Osterloh, M., Henning, Th., \& Launhardt, R. 1997, ApJS, 110, 71

Palla, F. \& Stahler, S.W. 1993, ApJ 418, 414

Phillips, C.J., Norris, R.P., Ellingsen, S.P., \& McCulloch, P.M. 1998, MNRAS 300, 1131

Sandell, G., Wright, M., \& Forster, J.R. 2003, ApJ 590, L45

Sargent, A.I. \& Beckwith, S. ApJ, 323, 294

Schreyer, K., Henning, Th., van der Tak, F.F.S., Boonman, A.M.S., \& van Dishoeck, E.F. 2002, A\&A, 394, 561

Sako, S., et al. 2005, Nature, 434, 995

Shepherd, D.S. \& Churchwell, E. 1996, ApJ, 472, 225

Shepherd, D.S., Yu, K.C., Bally, J., \& Testi, L. 2000, ApJ, 535, 833

Shepherd, D.S. \& Kurtz, S. 1999, ApJ 523, 690

Shepherd, D.S., Claussen, M.J., \& Kurtz, S. 2001, Science, 292, 1513

Shu, F.H., Adams, F.C., \& Lizano, S. 1987, ARAA, 25, 23

Shu, F.H., Najita, J.R., Shang, H., \& Li, Z.-Y., in Protostars and Planets IV, (Book - Tucson: University of Arizona Press; eds Mannings, V., Boss, A.P., Russell, S.S.), 2000, p. 789

Shu, F.H., Laughlin, G., Lizano, S., \& Galli, D. 2000, ApJ, 535, 190

Snell, R.L., Huang, Y.-L., Dickman, R.L., \& Claussen, M.J. 1988, ApJ, 325, 853

Snell, R.L., Dickman, R.L., \& Huang, Y.-L. 1990, ApJ, 352, 139

Sollins, P.K., Zhang, Q., Keto, E., \& Ho, P.T.P. 2005a, ApJ, 624, L49

Sollins, P.K., Zhang, Q., Keto, E., \& Ho, P.T.P. 2005b, ApJ, accepted

Sollins, P.K. \& Ho, P.T.P. 2005, ApJ, accepted

Stahler, S., Shu, F.S., \& Taam, R. 1980, ApJ, 241, 637

Thum, C., Matthews, H.E., Martín-Pintado, J., Serabyn, E., Planesas, P., \& Bachiller, R. 1994, A\&A, 215, 283

Tofani, G., Felli, M., Taylor, G.B., \& Hunter, T.R. 1995 A\&AS, 112, 229

Torrelles, J.M., Gómez, J.F., Garay, G., Rodríguez, L.F., Curiel, S., Cohen, R.J., \& Ho, P.T.P. 1998, ApJ, 509, 262

Torrelles, J.M., Patel, N.A., Gomez, J.F., Ho, P.T.P., Rodríguez, L.F., Anglada, G., Garay, G., Greenhill, L., Curiel, Salvador, \& Cantó, J. 2001, ApJ, 560, 853

Walsh, A.J., Burton, M.G., Hyland, A.R., \& Robinson, G. 1998, MNRAS, 301, 640

Wilking, B.A., Mundy, L.G., Blackwell, J.H., \& Howe, J.E. 1989, ApJ, 345, 257

Wilking, B.A., Blackwell, J.H., \& Mundy, L.G. 1990, AJ, 100, 758

Wolfire, M.G. \& Cassinelli, J.P. 1987 ApJ, 319, 850

Wood, D. O. S. \& Churchwell, E. 1989, ApJS, 69, 831

Yorke, H.W. \& Sonnhalter, C. 2002, ApJ 569, 846

Yorke, H.W. \& Krügel, E. 1977, A\&A, 54, 183

Zhang, Q., Hunter, T.R., \& Sridharan, T.K. 1998, ApJ, 505, L151

Zhang, Q., Hunter, T.R., Sridharan, T.K., \& Cesaroni, R. 1999, ApJ, 527, L117

Zhang, Q., Hunter, T.R., Sridharan, T.K., \& Ho, P.T.P. 2002, ApJ 566, 982

Zhang, Q., Hunter, T.K., Brand, J., Sridharan, T. K., Molinari, S., Kramer, M.A., \& Cesaroni, R. 2001, ApJ, 552, L167

Zhang, Q., Hunter, T.K., Brand, J., Sridharan, T. K., Cesaroni, R., Molinari, S., Wang, J., \& Kramer, M.A. 2005, ApJ, 625, 864 